\title{
Down Syndrome Preleukemia and Leukemia
}

\author{
Kelly W. Maloney, M.D. \\ Children's Hospital Colorado \\ Center for Cancer \& Blood Disorders \\ Aurora, Colorado
}

Jeffrey W. Taub, M.D.

Division of Pediatric Hematology/Oncology, Children's Hospital of Michigan,

Wayne State University School of Medicine, Detroit, Michigan

Yaddanapudi Ravindranath, M.B.;B.S.

Division of Pediatric Hematology/Oncology,

Children's Hospital of Michigan,

Wayne State University School of Medicine, Detroit, Michigan

Irene Roberts, M.D.

Centre for Haematology Commonwealth Building, Hammersmith Campus Imperial College London, London W12 0NN, United Kingdom

Paresh Vyas, M.D.

MRC Molecular Haematology Unit, Weatherall Institute of Molecular Medicine, University of Oxford and Department of Haematology, Oxford University Hospitals NHS Trust, OX3 9DS Oxford, United Kingdom 


\section{Introduction}

For over 160 years, Down syndrome (DS) has been linked to the English physician John Langdon Down. His essay published in 1866 "Observations on an ethnic classification of idiots", described a group of cognitively impaired individuals with common physical features. DS is now recognized as the most common chromosomal abnormality occurring in 1 in every 800 to 1000 live births. Neonates, children and adults with DS develop multiple medical disorders; hematologic disorders being one of the most well known. It has long been recognized that children with constitutional trisomy 21 (Down syndrome; DS) have a markedly increased risk of Acute Leukemia. The first description of a child with DS who developed Acute Leukemia was published in 1930. Subsequently, a national survey in the United States, provided support to the notion that children with DS had an increased risk of developing Leukemia. ${ }^{1}$ Remarkably, children with DS are at an increased risk both of Acute megakaryocyte-erythroid Leukaemia (known as Myeloid Leukemia of Down Syndrome - ML-DS) by 150-fold and of Acute B-lineage Lymphoblastic Leukaemia (BALL) by 33-fold compared to children without DS.

In ML-DS it is now clear that the initial event is perturbation of fetal hemopoiesis by Trisomy 21 (T21) itself. This leads to complex defects in fetal hemopoiesis and newborn hematology. In up to $28 \%$ of fetuses/newborns with DS, hemopoietic cells acquire mutations in the gene encoding the key megakaryocyte-erythroid transcription factor GATA1. Acquisition of GATA1 mutations can either be clinically silent or result in a clinically important preleukemic fetal/neonatal disorder Transient Abnormal Myelopoiesis (TAM). Most cases of TAM resolve without long-term clinical sequelae but in a proportion of cases with TAM, neonates/young children acquire additional genetic mutations that immortalize the TAM clone and result in frank ML-DS. There are parallel defects in DS fetal B-cell lymphopoiesis due to T21 that most likely results in acquisition of a series of cooperating and transforming mutations in genes encoding key regulators of B-lymphopoiesis (e.g. $J A K 2$ and $C L R F 2$ )

Thus, the unique features of DS-associated leukaemias arise because of the crucial role played by Trisomy 21 (T21) that then creates the right cellular and molecular environment for the acquisition of additional genetic mutations that together lead to Acute Leukemia. Thus, DS-associated leukemias represent potentially one of the most tractable human models to understand the biological basis of multistep leukaemogenesis and the impact of aneuploidy on cancer. Below, we highlight some of the recent clinical and biologic advances in these preleukemic and leukemic conditions.

\section{Key Points}

1) Down Syndrome children manifest multiple hematologic manifestations ranging from a) transient myeloproloferative disorder (TMD)/transient leukemia(TAM) at birth; b) acute myeloid leukemia (ML-DS) and c) Acute lypmphoblastic Leukemia (ALL)

2) Underlying primary basis for the varied hematologic manifestations is linked to gene dosage effect of Chr 21 encoded genes

3) TMD/TAM and ML-DS are characterized by the presence of truncating mutations in exon 2 of the hematopoietic transcription factor GATA1. Spontaneous resolutions is common in TMD/TAM and ML-DS is highly responsive to chemotherapy with resultant high cure rates compared to AML in Non-DS children

4) DS-ALL is characterized by the presence of mutations in JAK2 tyrosine kinase and IKZL1. In contrast to high cure rates in ML-DS, the results in DS-ALL are same are inferior to Non DS ALL in part due to the lower frequency of good response ALL subtypes and also the higher systemic toxicity of agents used in DS children.

\section{Trisomy 21 and human fetal haematopoiesis}


Fetal origin of Trisomy 21-associated leukaemias: Human T21 itself perturbs second trimester fetal liver haemopoietic stem/progenitor (HSPC) function. ${ }^{2-4}$ T21 increases the frequency of immunophenotypic hemopoietic stem cell (HSC) that have a biased erythroid-megakaryocyte primed gene expression profile compared with disomic HSC. Furthermore, multiple HSPC populations show increased megakaryocyte-erythroid output in colony assays. Coupled with this, there is an expansion of megakaryocyte-erythroid progenitors themselves. Consistent with this increased megakaryocyte output, immunohistochemical studies of T21 fetal liver sections show increased megakaryocyte numbers. However, megakaryocyte differentiation may be compromised as fetal liver megakaryocytes are morphologically abnormal. Corroborating data from human T21 embryonic stem cells (hES) and induced pluripotent stem cells (iPS) show increased erythroid and possible megakaryocyte production. ${ }^{5,6}$ In addition to increased, but likely perturbed megakaryocyteerythroid differentiation, there is a severe impairment of B-lymphoid development in DS fetal liver; with a $\sim 10$-fold reduction in pre-pro B-cells and B-cell potential of $\mathrm{HSC}$, in tandem with reduced HSC lymphoid gene expression priming. ${ }^{4}$

Abnormalities of blood counts in newborns with DS: Fetal liver hemopoietic defects most likely result in multi-lineage blood count changes in newborns with DS. These were initially noted in several small retrospective studies ${ }^{7-9}$, and more recently in a recent large prospective study. ${ }^{10}$ Neonates with DS had higher haemoglobin concentrations, increased circulating erythroblasts and abnormal red cell morphology, including macrocytosis, target cells and basophilic stippling. Median platelet counts were also lower than normal in neonates with DS. Thrombocytopenia was common and, although the median mean platelet volume was similar to neonates without DS, platelet morphology was abnormal (giant platelets, circulating megakaryocytes and/or megakaryocyte fragments) in $>95 \%$ of cases. Interestingly, neonates with DS had higher numbers of granulocytes and monocytes despite the reduction in granulocyte-monocyte progenitors in fetal liver. ${ }^{4}$ This may reflect a greater reliance on bone marrow haematopoiesis in late gestation and after birth. The total lymphocyte count (and particularly B-lymphocyte count) was reduced in the DS neonates.

Molecular basis for perturbed fetal/neonatal haematology due to T21: This is still largely unclear. The complex HSPC and peripheral defects suggest multiple genes are likely to be involved at distinct fetal stages of haematopoiesis. These genes could include the $\sim 300$ protein- and RNAencoding on chromosome 21 through gene dosage and/or more global impact on disomic gene regulation. At least two approaches have been used to identify genes linked to increased leukaemia susceptibility. First, studies in rare patients and mouse models have tried to narrow down the anatomical region of chromosome 21 responsible for the haemopoietic phenotype. Occasional patients with partial trisomy suggest that leukaemia risk is confined to a $8.5 \mathrm{Mb}$ region on chromosome 21; although conclusions here are limited by the very small number of cases of leukemia identified in these patients. ${ }^{11}$ Study of murine models trisomic for a variable number of human chromosome 21 genes have provided some insight, but are somewhat limited as none fully recapitulates the human hemopoietic phenotype in fetal and neonatal human DS. ${ }^{12-16}$ In summary overexpression of genes ERG, DYRK1A, CHAFIB and HLCS have all been implicated in a late onset adult myeloproliferative disorder and in facilitating the transformation to Acute Myeloid Leukemia. Second, analysis of gene expression in primary T21 fetal liver samples and hemopoietic cells derived from human T21 embryonic stem cells or induced pluripotent cells suggest that increased expression of several genes may contribute to deregulated hemopoiesis. These include: RUNX1, BACH1, ETS2, ERG, DYRK1A, GABPA and SON. However, careful functional experiments will be central to understanding if altered expression of any gene(s) is causal to the T21 hemopoietic phenotype.

In addition, it is unclear if the T21 phenotype is hemopoietic cell autonomous or is, in part, mediated by cells constituting the hemopoietic microenvironment. Several lines of indirect evidence suggest that fetal liver may provide the specialized microenvironment necessary for instigating and/or maintaining abnormal haematopoiesis in DS. First, as mentioned above, the observation that abnormal hemopoiesis occurs in fetal life suggest a yolk sac/fetal fetal microenvironment is 
important. Second, differential production and responsiveness of fetal tissues, including haematopoietic cells, to insulin growth factors (IGFs) is one of the few consistently reported differences between adult and fetal haematopoiesis. ${ }^{17-19}$

\section{Transient Abnormal Myelopoiesis (TAM) - Myeloid Preleukaemia of DS}

Clinical Findings: Though TAM is usually seen in newborns with DS, 7-16\% of TAM cases have mosaic T21. TAM can also present as hydrops fetalis with hepatosplenomegaly. The current definition of TAM is imprecise. It is defined as the presence of circulating blood blasts in a baby with typical clinical features of TAM who may, or may not, have hematologic abnormalities. Previous studies have used differing clinical and/or hematologic criteria to define TAM ${ }^{20}$. At one end of the spectrum TAM is detected as an incidental finding on review of a blood smear in an otherwise well baby. Often there will be an associated mild leucocytosis and thrombocytopenia. At the other of the spectrum, babies can be extremely sick with bruising, ascites, jaundice, hepatosplenomegaly, respiratory distress associated with pleural effusions and a low cardiac output state associated with a pericardial effusion. In one series $25 \%$ of the babies with TAM were referred to an intensive care unit. ${ }^{21}$

TAM - Laboratory Findings Laboratory tests (reviewed in ${ }^{22}$ can show leucocytosis (20-30\% of cases with a mean white cell count of $20-40 \times 10^{9} / \mathrm{L}$ with occasional cases having a white count of $\left.>100 \times 10^{9} / \mathrm{L}\right)$ though it can be normal on occasion. There is variable thrombocytopenia in $\sim 40 \%$ of cases (mean 80-100x $10^{9} / \mathrm{L}$ ) and occasionally severe anaemia (haemoglobin $<8 \mathrm{~g} / \mathrm{dl}$ ). Basophilia and an eosinophilia are often seen. Blasts can have the appearance of megakaryoblasts (with cytoplasmic "blebbing") but can also be featureless. Flow cytometry of blasts is helpful as they express CD34, CD38, CD117, CD7, CD56, CD33, CD45, the erythroid antigen CD235 and the megakaryocyte antigens CD41, CD42b and CD61. The bone marrow aspirate is not often helpful. The cellularity can be increased, normal or decreased. There is a deranged coagulation profile in 20$25 \%$ of cases with disseminated intravascular coagulation (DIC) in $\sim 7-10 \%$ of cases. Hepatic dysfunction is usually associated with hyperbilirubinemia, ascites, elevated transaminases and hepatic infiltration with blasts. In more severe cases there is hepatic fibrosis.

GATA1 mutation, definition of TAM and silent TAM and identification of children at risk of ML-DS: A diagnostic test in nearly all babies with TAM is the presence of acquired N-terminal truncating mutations ${ }^{23-27}$ and, more rarely internal in frame deletions, ${ }^{28}$ within exon 2 and, occasionally exon 3 , in the gene encoding the megakaryocyte-erythroid transcription factor GATA1. GATA1 mutations are required for TAM. The detection of N-terminal truncating GATAl mutations also extends to cases of DS mosaicism. There is an unique association these types of GATAl mutation and leukemogenesis in hemopoietic cells with T21. Though rare families and individuals have been described with N-terminal truncating GATAl mutations in non-DS individuals there is no documented increased risk of Acute Myeloid Leukemia in these cases. Non-DS patients with Nterminal truncating GATAl mutations present with a variety of haematologic phenotypes including multiple cytopenias ${ }^{29}$ and Diamond Blackfan Anemia phenotype ${ }^{30}$

The incidence of TAM has been the subject of debate. Retrospective studies suggest TAM affects $\sim 10 \%$ of DS neonates but these studies have used differing clinical and/or hematologic criteria to define TAM, none of which is specific for TAM. ${ }^{20,21,31}$ Importantly, no retrospective studies have systematically screened neonates for GATA1 mutations, a genetic marker specific for TAM and ML-DS. Thus, current definitions of TAM neither specify the percentage of blasts considered abnormal in DS neonates nor the role of GATA1 mutation analysis in the diagnosis. As a result, asymptomatic TAM may be missed in some neonates as blood counts and smears are often not performed. While in others babies, TAM may be over-diagnosed by relying on non-specific clinical and hematologic features. This possibility is borne out by the only large systematic GATAl mutation screen, performed by Sanger sequencing of genomic DNA extracted from dried blood spots, which found a prevalence of GATAl mutations in DS neonates of only $3.8 \%{ }^{32}$, in contrast to the $5-10 \%$ prevalence of TAM diagnosed by clinical and hematologic criteria (see review by ${ }^{22}$ ). 
The perception of the incidence and definition of TAM has been changed by a recent prospective study that systematically documented clinical features, blood counts, blood smears and GATA1 mutation in a large population-based cohort of newborns with DS. ${ }^{10}$ Surprisingly, 195/200 (97.5\%) DS neonates had circulating blasts (range: $1-77 \%$ ). In $\sim 8.5 \%$ of cases the blast count was $>10 \%$, a GATA1 mutation was detected by conventional Sanger sequencing and mutation detection techniques (Direct High Pressure Liquid Chromatography). In a further $20 \%$ of babies with blast counts $<10 \%$, GATA1 mutations were detected by the more sensitive next generation sequencing (NGS) mutation detection explaining, in part, the very frequent finding of blasts. ${ }^{10}$ No clinical or hematologic features distinguished them from GATA1 mutation-negative DS neonates or cases of TAM. Thus, GATA1 mutations are common, occur in $~ 28 \%$ of all neonates with DS but are often unsuspected and detectable only with sensitive methods. We suggest TAM is defined as blasts $>10 \%$ and a GATAl mutation detected by conventional sequencing and/or DHPLC. We suggest the term 'silent TAM' for those with a GATAl mutation detectable only by more sensitive sequencing techniques. In our series both TAM and silent TAM can transform to ML-DS. Longer-term followup is required to determine rates of transformation. To diagnose silent TAM and TAM, an initial evaluation of blood smears as well as full blood counts, as recommended by the American Academy of Pediatrics, ${ }^{33}$ is a useful, immediate screening step to identify DS neonates with "classical" TAM who may require early treatment (especially where GATAl analysis is unavailable or delayed). If possible, all DS neonates should also have GATA1 mutation analysis by Sanger sequencing and DHPLC to quickly identify those with large mutant GATAl clones. For DS neonates without mutations by Sanger sequencing or DHPLC, next generation re-sequencing can be used to identify those with small mutant GATAl clones. By comprehensively detecting GATAl mutations, pediatric hematology follow-up can be limited to those at risk of transformation rather than all babies with blasts (i.e. all babies with DS).

Outcome and management of TAM: In most babies (80-90\%) the condition only requires observation and blood count monitoring as it resolves spontaneously over a 1-3 month period (median time to resolution 47 days $^{31}$ ). However, a proportion of babies require therapy for clinical symptoms e.g. cardiac failure or DIC. However, it is unclear which infants will benefit from treatment and what the most effective treatment is. Accordingly treatment policies vary considerably between centres. Both the US Pediatric/Children's Oncology Group (POG/COG) and the German I-BFM group have developed treatment guidelines for TAM that can be instituted at the physician's discretion. ${ }^{21,31}$

As TAM blasts are highly sensitive to cytarabine, it is the basis of cytoreductive therapy. Response is generally rapid with disappearance of peripheral blasts within 7 days of treatment. However, in babies with severe liver disease associated with fibrosis the response to chemotherapy is poor. In the POG study 9481, cytosine arabinoside was given either as $10 \mathrm{mg} / \mathrm{m}^{2} / \mathrm{dose}$ or $1.2-1.5 \mathrm{mg} / \mathrm{kg} / \mathrm{dose}$ subcutaneously or intravenously by slow injection twice a day for 7 days. ${ }^{20}$ In the AML-BFM study, $0.5-1.5 \mathrm{mg} / \mathrm{kg}$ was administered for $3-12$ days $^{21}$. The higher dose of cytarabine (3.33 $\mathrm{mg} / \mathrm{kg} / 24$ hours given by continuous infusion for 5 days) used in the COG A2971 study is probably not required and was associated with significant toxicity. ${ }^{31}$ Despite therapy, 10-20\% of babies die of TAM within 3 months with hepatic and renal failure and DIC. These babies often present with a higher white count (>100x10\%/L), ascites, effusions, coagulopathy and develop hepatic fibrosis.

Transformation to ML-DS: Following the clinical resolution of TAM with normalization of blood counts either spontaneously or after treatment with very low-dose cytarabine, up to $30 \%$ of these neonates with clinical TAM will subsequently develop ML-DS. ${ }^{34}$ In a baby with a history of TAM often the same GATAl mutation is detected when the child presents with ML-DS proving that the two disorders are clonally linked. ${ }^{24,25,27}$ In $\sim 15-25 \%$ cases multiple GATA1 mutations are detected in both TAM and ML-DS suggesting that multiple GATA1 mutant clones exist. ${ }^{27}$

Clinical Features of $M L-D S$ : ML-DS Children with DS represent approximately $15 \%$ of pediatric AML cases. ${ }^{35}$ Reports from both the United Kingdom Childhood Cancer Study and the Children's Cancer Group (CCG) identified that the mean age at diagnosis of ML-DS was lower than the age 
for children without DS [2.2 years versus 6.7 years and 1.8 versus 7.5 years, respectively] ${ }^{35,36}$. Overall, 95\% of ML-DS cases are diagnosed before the age of 4 years. ${ }^{37}$ Progression to ML-DS does not occur in children beyond the age of 5 years old. ${ }^{37}$

Progression to ML-DS occurs at a variable tempo. In some children progression is heralded by a period of falling blood counts, especially thrombocytopenia and leucopenia. Cytopenia may be short or prolonged over months prior to formal diagnosis of ML-DS. It is often associated with dysplastic changes in peripheral blood cells. At presentation of ML-DS, children with DS may not appear acutely ill compared to children without DS. This is due, in part, from closer clinical monitoring of these children based on their known increased risk to develop leukemia and early evidence of abnormalities in blood count parameters indicating evidence of potential progression to leukemia in the absence of clinical symptoms. These early signs of myelodysplasia, are characterized by progressive anemia and thrombocytopenia in association with dysplastic erythroid cells and megakaryocytes in the bone marrow. ${ }^{38}$ This myelodysplastic phase frequently precedes the development of AML and the spectrum of both myelodysplastic syndrome (MDS) and AML have been known collectively as myeloid leukemia of DS (ML-DS). ${ }^{38,39}$ Rarely ML-DS can progress directly from aggressive TAM. In these cases the diagnosis is problematic as it is difficult to distinguish between TAM that is not resolving and development of ML-DS. A bone marrow aspirate and trephine can be helpful to confirm $>30 \%$ blasts. Often the blast count is lower as the marrow sample on aspirate is haemodilute. In these cases the trephine shows marrow fibrosis echoing the liver fibrosis seen in some cases of TAM. If the marrow is aspirable or if there are circulating blasts, flow cytometry is valuable as blasts express the same cell surface markers as TAM blasts.

Laboratory Features of ML-DS: Several other distinctive differences exist in the biological features of ML-DS compared to AML in children without DS. Amongst the 8 different French-AmericanBritish (FAB) subtypes of AML, children with DS have a disproportionately high proportion with the acute megakaryocytic/megakaryoblastic (AMKL, M7) phenotype. Zipursky estimated that DS children have a 500-fold increased risk of developing ML-DS compared to children without DS, with some clinical studies identifying the ML-DS phenotype in over $90 \%$ of ML-DS cases. ${ }^{38}$ Besides the morphologic identification of the ML-DS phenotype, megakaryoblasts are identified by their immunophenotype expression of the platelet-associated membrane antigens (glycoprotein IIb/IIIa) using CD41/61 antibodies and frequently the aberrant expression of the T-cell antigen, CD7, and the thrombospondin receptor, CD36. ${ }^{40,41}$. High expression of CD36 marker distinguishes ML-DS from most cases of de novo AMKL and may be a surrogate for the developmental stage of the megakryoblasts identified by molecular studies. ${ }^{18,19,41}$

The proportion of DS patients who fit the criteria of MDS based on the bone marrow involvement containing less that $30 \%$ blasts, was approximately $30 \%$ for both the Children's Oncology Group A2971 and CCG2891 studies. ${ }^{35,42}$ Structural chromosomal abnormalities detected in ML-DS include trisomy 8, trisomy 11 and trisomy 21 (besides the constitutional trisomy 21); dup(1p), $\operatorname{del}(6 q), \operatorname{del}(7 p), \operatorname{dup}(7 q)$ and $\operatorname{del}(16 q) .{ }^{43}$ In contrast, the classic cytogenetic abnormalities seen in AML cases in children without DS including AML-ETO $\mathrm{t}(8 ; 21) ; P M L-R A R A \mathrm{t}(15 ; 17) ; C B F B-$ MYH11 inv(16), MLL 11q23 rearrangements are not seen in ML-DS. Furthermore, neonates and children with DS developing ML-DS usually do not acquire the specific non-DS childhood AMKL cytogenetic abnormality RBM15-MKL1 $\mathrm{t}(1 ; 22)^{44}$ or the cryptic inversion of chromosome 16 that produces the CBFA2T3-GLIS2 fusion gene. ${ }^{45,46}$

Molecular abnormalities in ML-DS: Whole genome/exome sequencing identified that the mean number of somatic mutations in TAM cases (with GATAl mutations in all cases) was 1.5 , while the number of mutations was significantly higher in ML-DS cases than in TAM. ${ }^{47,48}$. Acquired mutations were identified in a genes encoding components of the cohesin complex (RAD21, STAG2, NIPBL, SMC3, SMC1A); chromatin regulators (EZH2, SUZ12, ASXL1, DNMT3A); cytokine signaling pathway regulators (JAK1, JAK3, MPL, KRAS, NRAS, PTPN11, SH2B3) and other genes that have also been previously shown to be been mutated in leukemia (TP53, ETV6, 
SRSF2). These observations indicate that TAM is caused by a GATA1 mutation together with trisomy 21 and progresses to ML-DS due to the acquisition of additional mutations. ${ }^{48}$

\section{Therapy of ML-DS}

Although the chemotherapy agents used in the treatment of ML-DS are the same as for children without DS, current treatment strategies differ significantly between the DS and non-DS patient groups and in particular, balancing curative therapy against the risk of treatment-associated morbidity and mortality for children with DS. The Pediatric Oncology Group 8498 trial was the first to identify that ML-DS had very high cure rates when children with DS were treated with equivalent protocols as for children without DS. ${ }^{49}$ Previous to this report, less than optimal leukemia therapy for children with DS appeared to be a significant factor in their poor survival rates, due to a perception that DS children would not tolerate contemporary AML therapies. Subsequent studies from CCG, POG, Medical Research Council, Nordic Society of Pediatric/Hematology Oncology and the Berlin-Frankfurt-Munster (BFM) cooperative groups confirmed the high cure rates for ML-DS with overall event-free survival rates of approximately 80\%. ${ }^{50-54}$ The utilization of high-dose cytosine arabinoside (araC)-based therapy for ML-DS patients, appeared to have contributed to the improved survival rates. ${ }^{55}$

The time-intensive strategy for treating pediatric AML (without evidence of full marrow recovery from neutropenia and thrombocytopenia), was found to be too toxic for ML-DS patients as highlighted by the CCG 2861/2891 studies, in which there was a 32\% treatment-associated mortality rate for patients treated on the intensive-timed treatment arm. ${ }^{52}$ The MRC AM10/12 studies reported that children with DS experienced a $27 \%$ treatment-related mortality rate who received equivalent drug dosing as for children without DS. ${ }^{54}$ Cardiotoxicity was a significant treatment-related complication for children with DS treated on POG 9421 (which used a high total cumulative anthracycline dose); $21 \%$ of children developed late onset congestive heart failure including several treatment-associated (and non-leukemic) deaths. ${ }^{56}$

The CCG-2891 study found that ML-DS patients experience similar toxicities compared to non-DS patients when a decreased dose-intensity regimen was administered. ${ }^{57}$ This has also led to the design of DS-specific AML protocols including COG A2971 ${ }^{42}$ and AAML0431.

In order to reduce therapy-associated toxicity, other groups have reported the use of either intermediate dose or very low-dose araC. Studies from Japan used an intermediate dose araC-based regimen [5 cycles of araC $100 \mathrm{mg} / \mathrm{m}^{2} /$ day x 7 days with an $83 \%$ EFS rate ${ }^{58}$ Canadian studies have utilized repetitive courses of very low-dose araC $\left(10 \mathrm{mg} / \mathrm{m}^{2} / \mathrm{dose}\right)$, with several non-responsive patients being able to be salvaged with intensive AML therapies. ${ }^{59}$ A study of ML-DS patients treated in France from 1990-2003, however reported that low-dose chemotherapy regimens including araC were inferior compared to standard-dose chemotherapy regimens. ${ }^{60}$

The current COG AAML0431 trial is investigating the clinical significance of MRD testing for ML-DS, which may identify patients who could be treated with reduced intensity therapy.

\section{Prognostic Factors}

Several prognostic factors have been reported for ML-DS patients, which are associated with outcome. The BFM group identified that a prior diagnosis of TMD was associated with superior EFS rates compared to patients without a TMD history, ${ }^{21}$ though the results of the COG studies reported equivalent outcomes. ${ }^{42,57}$ ML-DS patients older than 4 years of age (who comprise $\sim 5 \%$ of the patient group) have a poor outcome; for the CCG-2891 study, EFS rates were only 33\%.

${ }^{57}$ This may indicate differences in leukemia biology amongst DS patients including a low detection rate of GATA1 mutations in blast cells amongst the older patients. ${ }^{61}$

An international retrospective analysis of 451 ML-DS patients had an overall survival rate of $79 \%$ and identified several factors: i) patients with a normal karyotypes had an overall relapse rate of $21 \%$ compared to $9 \%$ with patients with an aberrant karyotype, ii) patients with a white blood cell count $\geq 20,000 / \mu \mathrm{L}$ and age $>3$ years were independent predictors for poor EFS. ML-DS patients with monosomy 7 had a moderately worse outcome with EFS rates of $69 \%$, though still superior than monosomy 7 AML in children without DS. ${ }^{62}$ 
Although the ML-DS group overall, has an excellent prognosis, a minority of patients with either refractory or relapsed disease have a poor prognosis. DS patients with relapsed leukemia treated on the POG 9421 and CCG-2891 AML studies had an overall survival (OS) rate of only $12 \% .{ }^{63}$ Twenty six Japanese DS AML patients (including 22 with ML-DS) with relapsed or refractory disease had an OS rate of only $25.9 \% .{ }^{64}$ In another study, ML-DS patients only had a $19 \%$ probability of survival following stem cell transplant (SCT). These studies all highlight that DS patients with refractory/relapsed leukemia have very chemotherapy resistant disease. A recent Japanese study reported an $80 \%$ EFS rate for ML-DS using a lower-intensity conditioning regimen preceding SCT for ML-DS ${ }^{65}$ though the inclusion of patients in first remission who may have already been cured by frontline chemotherapy, may have biased the very favorable results.

Several new therapeutic approaches may offer promising treatments for relapsed ML-DS cases: i)Preventing cell cycle checkpoint activation by inhibiting the upstream kinase weel with the inhibitor MK-1775 in combination with araC. MK-1775 was able to synergistically enhance araC cytotoxic effects in ML-DS cell lines and ex vivo patient samples by abrogation of an intra-S phase DNA damage checkpoint and enhancement of araC-induced DNA damage. ${ }^{66}$ ii)Inhibition of aurora A kinase with MLN9237 was able to induce polyploidization and differentiation of non-DS ML-DS cells and a ML-DS cell line. ${ }^{67}$ iii)Histone deacetylase inhibitors inducing apoptosis by suppressing autophagy. ${ }^{68}$

\section{ACUTE LYMPHOBLASTIC LEUKEMIA IN DOWN SYNDROME:}

\section{INCIDENCE:}

Studies suggest a 10-20 fold increased risk of leukemia in children with Down syndrome (DS), with acute lymphoblastic leukemia (ALL) occurring in 1 in 300 children with DS vs. 1 in 3500 children without DS. ${ }^{69,70}$ Hasle et al described the incidence of malignancy in 2,814 individuals with DS registered in the Danish Cytogenetic Registry during the period 1968-1995 and reported that leukemia made up $60 \%$ of the malignant diseases overall, with the majority occurring prior to age 15 years. ${ }^{71}$ In this registry study of individuals with DS, no cases of leukemia were seen after the age of 29 years.

\section{CLINICAL FEATURES, THERAPY AND OUTCOME IN CHILDREN WITH DS ALL:}

Children with DS and ALL do have a few unique clinical characteristics as compared to those without DS as well as more therapy related challenges. The age distribution at presentation of the leukemia is similar in children with and without DS, with the majority of children presenting at age $>1$ year. ALL is very rarely diagnosed in DS children who are less than one year of age whereas approximately $2.6 \%$ of non-DS ALL cases occur in infants less than one year of age. ${ }^{72-76}$ Most studies also report no significant difference in initial white blood cell (WBC) count, racial or ethnic differences in the population or assignment to National Cancer Institute (NCI) risk groups ${ }^{72,73,75-79}$. Although no uniformly reported, the incidence of a mediastinal mass or the presence of central nervous system (CNS) disease at diagnosis is similar between DS ALL and non-DS ALL ${ }^{72,73,78}$. However, there are clear immunophenotypic differences between DS and non-DS ALL. T-cell phenotype (T-ALL) and mature B-cell phenotype are very rare in patients with DS but occur at higher incidences in non-DS leukemia. ${ }^{75}$ A recent review of 708 DS ALL patients found only 5 DS patients with T-ALL, compared to the expected $10-15 \%$ in non-DS ALL. ${ }^{80}$

Induction therapy for DS ALL and non-DS ALL is now essentially the same with only minimal differences in DS ALL to enhance safety in the majority of treatment protocols. In most modern therapeutic trials, clinical remission (CR) at the end of induction is found to be similar between DS ALL and non-DS ALL. ${ }^{72,73,79}$ The rates of CR at end induction are reported to range from $96 \%$ 99\% and were not statistically significant from the CR rates of non-DS ALL patients in these studies. $^{75,77,81}$ However, a large retrospective study evaluated 653 DS ALL patients and found induction failures to occur in $3 \%$ of DS ALL vs $1 \%$ of non-DS ALL $(\mathrm{p}<0.001) .{ }^{80}$ Increased 
mortality, primarily due to infection, during remission induction in DS ALL has been well described and contributes to induction failure. ${ }^{80,82,83}$

Additional measures about the depth of remission or rapidity of response to induction therapy are used to augment the risk classification and prognosis. There is limited data that suggests DS ALL responses in small studies are equivalent to those with non-DS ALL. Prednisone response (prednisone response $=$ number of lymphoblasts in blood after a 7-day exposure to prednisone with a good response being $<1000 / \mu$ blood blasts) was not different between DS ALL and non-DS ALL in the Berlin Frankfurt Munster protocols (BFM) with prednisone good response in $90.7 \%$ and $98.1 \%$, respectively. ${ }^{73}$ Levels of bone marrow minimal residual disease (MRD) as measured by flow cytometry (measured from $<0.1 \%$ to $>1 \%$ ) did not show a difference between DS-ALL vs. non-DS ALL at day 29 of induction in the most recent Children's Oncology Group (COG) therapeutic trials. ${ }^{84}$ In addition, the corresponding marrow response at day 8 and day 15 was similar between DS ALL and non-DS ALL in this study.

Currently, the majority of ALL cooperative group protocols use the same or very similar therapy for DS ALL as for their patients without DS and have in some cases successfully increased the intensity of therapy. ${ }^{72,73,77,85}$ (L. Silverman, personal communication). Frequently, however, there may be additional modifications or supportive care guidelines for DS ALL vs non DS ALL. COG and Medical Research Council United Kingdom Childhood Acute Lymphoblastic Leukaemia (MRC UKALL) trials now incorporate discontinuous dexamethasone during delayed intensification. Additionally, the COG therapy trials added leucovorin rescue after intrathecal administration of methotrexate. ${ }^{82,83}$ Anthracycline exposure in DS ALL is approached differently amongst the groups. Two groups are decreasing the anthracycline exposure to DS ALL high risk patients by only giving induction daunomycin to those patients with a slow response. ${ }^{86}$ However, St. Jude Research Hospital ALL therapies incorporate anthracycline along with steroids in induction with no excess toxicity or mortality in the DS ALL population (M. Relling, personal communication). The balance in modern therapy is to continue to improve the survival of this special group of patients while decreasing the severe adverse effects of therapy in DS ALL. One of the key agents used in ALL therapy is methotrexate (MTX). It is well known that DS ALL patients are more susceptible to MTX-induced side effects than non-DS ALL patients. Multiple studies have shown that patients with DS ALL suffer significantly higher incidences of gastrointestinal toxicity primarily as well as more hematologic toxicity. ${ }^{80,87-90}$ For this reason, infusional $\mathrm{mtx}$ is often started at a more modest dose for DS patients, increasing if tolerated. Treatment related mortality (TRM) is higher for patients with DS ALL as compared to non-DS ALL. In the large retrospective study, the TRM for DS patients was $7.7 \%$ as comparted to $2.3 \%$ in non-DS patients. ${ }^{80}$ The majority of the TRM in DS ALL is due to infection/sepsis. In fact, the infection related mortality (IRM) can be as high as $18.6 \%$ vs $1.9 \%$ in non-DS ALL. ${ }^{82}$ Additionally, IRM in non-DS ALL occurs primarily during induction but for DS ALL, this risk is spread throughout all phases of therapy. ${ }^{80,82,91}$ When caring for DS ALL patients, careful observation and adherence to supportive care measures is quite important. However, further studies are needed to assess the value of additional supportive care such as prophylactic antibiotics and immunoglobin use.

The prognosis of children with DS ALL is worse than for those with non-DS ALL in general ${ }^{76,80,91}$. The Ponte di Legno study group reported a lower event free survival (EFS) of $64 \%$ vs $81 \%$ at 8 years for DS ALL vs non-DS ALL and consequently a lower overall survival (OS) of $74 \%$ vs $89 \%$, respectively. ${ }^{80}$ Similar results have also been show in smaller studies. COG reported an inferior EFS and OS for DS patients enrolled on the AALL0232, the most recently completed HR trial, largely due to increased toxic mortality rates as discussed above. The EFS for the DS patients on the NCI standard risk trial, AALL0331, were similar to non-DS patients but the OS was significantly lower, $89 \%$ vs $96 \%$, respectively. ${ }^{91}$

Despite a high rate of TRM, relapse is still a main cause of treatment failure in this group of patients. ${ }^{80}$ However, enrollment on relapse treatment protocols for this group of patients has not been consistent so there is limited date about the outcome following relapse. Relapses in DS ALL 
patients tend to occur later when compared to non-DS ALL. ${ }^{80,92}$ The BFM106529 relapsed trials (ALL REZ BFM) retrospectively found that a significantly higher proportion of children with DS has fatal treatment-related adverse events during induction therapy (22\%) and subsequent treatment phases (10\%) as compared to children without DS when treated for relapse (3\% and 6\%, respectively). In addition, hematopoietic stem cell transplant was used less frequently in DS ALL after relapse than in non-DS ALL. ${ }^{92}$ As the numbers are small, further understanding of tolerance of therapy and transplant in this population is important for making therapeutic decisions for relapse.

\section{BIOLOGIC FEATURES OF DS ALL:}

The biologic features of DS with ALL are distinctly different than ALL in children without DS. Additionally, DS ALL does not have an overriding lesion responsible for the transformation as does DS Acute Myeloid Leukemia. The Ponte di Legno study group reported $B C R-A B L 1$ fusion in $0.7 \%$ compared to $2.4 \%$ non-DS ALL and MLL rearrangements of $<1 \%$ in DS ALL vs $1.2 \%$ of nonDS ALL. ${ }^{80}$ These findings are consistent with other studies as well which have confirmed this lower incidence of hypodiploidy, $\mathrm{t}(9 ; 22)$ and 11q23 translocations in DS ALL as compared to nonDS ALL. ${ }^{43,78,84}$

Different frequencies of favorable subtypes in DS ALL may also be responsible for some of the inferior survival seen in DS ALL. COG 9900 series, a series of therapeutic trials with a uniform classification strategy, showed that the incidence of the favorable cytogenetic lesions are different between DS ALL and non-DS ALL. ${ }^{84}$ The incidence of ETV6-RUNX1 fusion transcripts were found in $2.5 \%$ of DS ALL vs. $24 \%$ of non-DS ALL. Similarly, trisomy of both chromosomes 4 and 10 occurred in $7.7 \%$ of DS ALL versus $23.9 \%$ of non-DS ALL. The proportion of high hyperdiploidy as measured by a DNA index of greater than 1.16 was significantly different between the two groups with $5 \%$ in DS ALL versus $24.6 \%$ in non-DS ALL.

Additional genetic abnormalities, JAK mutations, Cytokine receptor-like factor 2 (CRLF2) expression and $I K Z F 1$ mutations, have been recognized in pediatric ALL. The aberrant expression of $C R L F 2$ is detected in $50 \%$ of DS ALL vs $5 \%$ non-DS ALL. ${ }^{93}$ The majority of the CRLF2 alterations were observed in cases lacking translocations associated with ALL, suggesting that CFLF2 alteration is a potent leukemogenic event in the setting of trisomy $21 .^{93,94}$ Although the overexpression of $C R L F 2$ is frequently found in DS ALL, it has not been proven to be associated with a therapeutic outcome which is in contrast to non-DS ALL where CFLF2 overexpression has been associated with a poor EFS. ${ }^{94,95} J A K 2$ activating mutations have recently been described in approximately $20 \%$ of DS-ALL cases and approximately $10 \%$ of high risk non-DS ALL. ${ }^{96,97}$ DS ALL patients with JAK2 mutations have a similar outcome to those without the mutation. ${ }^{80,98}$ The identification of $J A K 2$ mutations in DS ALL leads to the possibility of targeted therapy with a JAK2 inhibitor. Early trials of these drugs are occurring in myeloproliferative disorders and may then lead to additional therapeutic options for DS ALL with potentially less toxicity. Recently, $I K Z F 1$ gene deletions/alterations have been shown to be associated with a very poor outcome in Bcell-progenitor ALL. IKZF1 deletions have been reported in $~ 30 \%$ of DS ALL patients. Similar to those with non-DS ALL, these mutations are associated with a poor prognosis, EFS $45 \%$ with DS ALL and IKZF1 mutation vs $95 \%$ for DS ALL without the mutation. ${ }^{99}$ Larger trials as we move forward will be needed to confirm the prognostic importance of these more newly described genetic alterations in DS ALL.

\section{Conclusion:}

Children with DS and ALL have unique biologic, cytogenetic and intrinsic factors which affect their treatment and outcome. As their EFS and OS are poorer than non-DS ALL, it is important to continue to study the biology of their leukemia, enroll them on therapeutic trials, including relapse trials, investigate new agents that could potentially improve their leukemia free survival without additional toxicity and strive to maximize the supportive care these patients need. 


\section{References}

1. Krivit W, Good RA. Simultaneous occurrence of mongolism and leukemia; report of a nationwide survey. AMA journal of diseases of children 1957;94:289-93.

2. Tunstall-Pedoe 0, Roy A, Karadimitris A, et al. Abnormalities in the myeloid compartment in Down Syndrome fetal liver precede acquisition of GATA1 mutations. Blood 2008;112:4507-11.

3. Chou ST, Opalinska JB, Yao Y, et al. Trisomy 21 enhances human fetal erythromegakaryocytic development. Blood 2008;112:4503-6.

4. Roy A, Cowan G, Mead AJ, et al. Perturbation of fetal liver hematopoietic stem and progenitor cell development by trisomy 21. Proceedings of the National Academy of Sciences of the United States of America 2012;109:17579-84.

5. Chou ST, Byrska-Bishop M, Tober JM, et al. Trisomy 21-associated defects in human primitive hematopoiesis revealed through induced pluripotent stem cells. Proceedings of the National Academy of Sciences of the United States of America 2012.

6. Maclean GA, Menne TF, Guo G, et al. Altered hematopoiesis in trisomy 21 as revealed through in vitro differentiation of isogenic human pluripotent cells. Proceedings of the National Academy of Sciences of the United States of America 2012.

7. Starc TJ. Erythrocyte macrocytosis in infants and children with Down syndrome. The Journal of pediatrics 1992;121:578-81.

8. Kivivuori SM, Rajantie J, Siimes MA. Peripheral blood cell counts in infants with Down's syndrome. Clin Genet 1996;49:15-9.

9. Henry E, Walker D, Wiedmeier SE, Christensen RD. Hematological abnormalities during the first week of life among neonates with Down syndrome: data from a multihospital healthcare system. Am J Med Genet A 2007;143:42-50.

10. Roberts I, Alford K, Hall G, et al. GATA1-mutant clones are frequent and often unsuspected in babies with Down syndrome: identification of a population at risk of leukemia. Blood 2013;122:3908-17.

11. Korbel JO, Tirosh-Wagner T, Urban AE, et al. The genetic architecture of Down syndrome phenotypes revealed by high-resolution analysis of human segmental trisomies. Proceedings of the National Academy of Sciences of the United States of America 2009;106:12031-6.

12. Alford KA, Slender A, Vanes L, et al. Perturbed hematopoiesis in the Tc1 mouse model of Down syndrome. Blood 2010;115:2928-37.

13. Kirsammer G, Jilani S, Liu H, et al. Highly penetrant myeloproliferative disease in the Ts65Dn mouse model of Down syndrome. Blood 2008;111:767-75.

14. Stankiewicz MJ, Crispino JD. ETS2 and ERG promote megakaryopoiesis and synergize with alterations in GATA-1 to immortalize hematopoietic progenitor cells. Blood 2009;113:3337-47.

15. Salek-Ardakani S, Smooha G, de Boer J, et al. ERG is a megakaryocytic oncogene. Cancer research 2009;69:4665-73.

16. Malinge S, Bliss-Moreau M, Kirsammer G, et al. Increased dosage of the chromosome 21 ortholog Dyrk1a promotes megakaryoblastic leukemia in a murine model of Down syndrome. The Journal of clinical investigation 2012;122:948-62.

17. Chou S, Lodish HF. Fetal liver hepatic progenitors are supportive stromal cells for hematopoietic stem cells. Proceedings of the National Academy of Sciences of the United States of America 2010;107:7799-804. 
18. Klusmann JH, Godinho FJ, Heitmann K, et al. Developmental stage-specific interplay of GATA1 and IGF signaling in fetal megakaryopoiesis and leukemogenesis. Genes \& development 2010;24:1659-72.

19. Bourquin JP, Subramanian A, Langebrake C, et al. Identification of distinct molecular phenotypes in acute megakaryoblastic leukemia by gene expression profiling. Proceedings of the National Academy of Sciences of the United States of America 2006;103:3339-44.

20. Massey GV, Zipursky A, Chang MN, et al. A prospective study of the natural history of transient leukemia (TL) in neonates with Down syndrome (DS): Children's Oncology Group (COG) study POG-9481. Blood 2006;107:4606-13.

21. Klusmann JH, Creutzig U, Zimmermann M, et al. Treatment and prognostic impact of transient leukemia in neonates with Down syndrome. Blood 2008;111:2991-8.

22. Roy A, Roberts I, Vyas P. Biology and management of transient abnormal myelopoiesis (TAM) in children with Down syndrome. Seminars in fetal \& neonatal medicine 2012;17:196201.

23. Wechsler J, Greene M, McDevitt MA, et al. Acquired mutations in GATA1 in the megakaryoblastic leukemia of Down syndrome. Nature genetics 2002;32:148-52.

24. Rainis L, Bercovich D, Strehl S, et al. Mutations in exon 2 of GATA1 are early events in megakaryocytic malignancies associated with trisomy 21. Blood 2003;102:981-6.

25. Hitzler JK, Cheung J, Li Y, Scherer SW, Zipursky A. GATA1 mutations in transient leukemia and acute megakaryoblastic leukemia of Down syndrome. Blood 2003;101:4301-4. 26. $\mathrm{Xu}$ G, Nagano M, Kanezaki R, et al. Frequent mutations in the GATA-1 gene in the transient myeloproliferative disorder of Down syndrome. Blood 2003;102:2960-8.

27. Ahmed M, Sternberg A, Hall G, et al. Natural history of GATA1 mutations in Down syndrome. Blood 2004;103:2480-9.

28. Toki T, Kanezaki R, Kobayashi E, et al. Naturally occurring oncogenic GATA1 mutants with internal deletions in transient abnormal myelopoiesis in Down syndrome. Blood 2013;121:3181-4.

29. Hollanda LM, Lima CS, Cunha AF, et al. An inherited mutation leading to production of only the short isoform of GATA-1 is associated with impaired erythropoiesis. Nature genetics 2006;38:807-12.

30. Sankaran VG, Ghazvinian R, Do R, et al. Exome sequencing identifies GATA1 mutations resulting in Diamond-Blackfan anemia. The Journal of clinical investigation 2012;122:243943.

31. Gamis AS, Alonzo TA, Gerbing RB, et al. Natural history of transient myeloproliferative disorder clinically diagnosed in Down syndrome neonates: a report from the Children's Oncology Group Study A2971. Blood 2011;118:6752-9; quiz 996.

32. Pine SR, Guo Q, Yin C, Jayabose S, Druschel CM, Sandoval C. Incidence and clinical implications of GATA1 mutations in newborns with Down syndrome. Blood 2007;110:212831.

33. Bull MJ. Health supervision for children with Down syndrome. Pediatrics 2011;128:393-406.

34. Zipursky A. Transient leukaemia - a benign form of leukaemia in newborn infants with trisomy 21. British journal of haematology 2003;120:930-8.

35. Gamis AS, Woods WG, Alonzo TA, et al. Increased age at diagnosis has a significantly negative effect on outcome in children with Down syndrome and acute myeloid leukemia: a report from the Children's Cancer Group Study 2891. Journal of clinical oncology : official journal of the American Society of Clinical Oncology 2003;21:3415-22.

36. James R, Lightfoot T, Simpson J, et al. Acute leukemia in children with Down's syndrome: the importance of population based study. Haematologica 2008;93:1262-3. 
37. Hasle H, Abrahamsson J, Arola M, et al. Myeloid leukemia in children 4 years or older with Down syndrome often lacks GATA1 mutation and cytogenetics and risk of relapse are more akin to sporadic AML. Leukemia 2007;22:1428-30.

38. Zipursky A, Thorner P, De Harven E, Christensen H, Doyle J. Myelodysplasia and acute megakaryoblastic leukemia in Down's syndrome. Leuk Res 1994;18:163-71.

39. Heerema-McKenney A, Arber DA. Acute myeloid leukemia. Hematology/oncology clinics of North America 2009;23:633-54.

40. Langebrake C, Creutzig U, Reinhardt D. Immunophenotype of Down syndrome acute myeloid leukemia and transient myeloproliferative disease differs significantly from other diseases with morphologically identical or similar blasts. Klin Padiatr 2005;217:126-34.

41. Savasan S, Buck S, Raimondi SC, et al. CD36 (thrombospondin receptor) expression in childhood acute megakaryoblastic leukemia: in vitro drug sensitivity and outcome. Leuk Lymphoma 2006;47:2076-83.

42. Sorrell AD, Alonzo TA, Hilden JM, et al. Favorable survival maintained in children who have myeloid leukemia associated with Down syndrome using reduced-dose chemotherapy on Children's Oncology Group trial A2971: a report from the Children's Oncology Group. Cancer 2012;118:4806-14.

43. Forestier E, Izraeli S, Beverloo B, et al. Cytogenetic features of acute lymphoblastic and myeloid leukemias in pediatric patients with Down syndrome: an iBFM-SG study. Blood 2008;111:1575-83.

44. Mercher T, Coniat MB, Monni R, et al. Involvement of a human gene related to the Drosophila spen gene in the recurrent $t(1 ; 22)$ translocation of acute megakaryocytic leukemia. Proceedings of the National Academy of Sciences of the United States of America 2001;98:5776-9.

45. Thiollier C, Lopez CK, Gerby B, et al. Characterization of novel genomic alterations and therapeutic approaches using acute megakaryoblastic leukemia xenograft models. The Journal of experimental medicine 2012;209:2017-31.

46. Gruber TA, Larson Gedman A, Zhang J, et al. An Inv(16)(p13.3q24.3)-encoded CBFA2T3-GLIS2 fusion protein defines an aggressive subtype of pediatric acute megakaryoblastic leukemia. Cancer cell 2012;22:683-97.

47. Yoshida K, Toki T, Okuno Y, et al. The landscape of somatic mutations in Down syndrome-related myeloid disorders. Nature genetics 2013;45:1293-9.

48. Nikolaev SI, Santoni F, Vannier A, et al. Exome sequencing identifies putative drivers of progression of transient myeloproliferative disorder to AMKL in infants with Down syndrome. Blood 2013;122:554-61.

49. Ravindranath Y, Abella E, Krischer JP, et al. Acute myeloid leukemia (AML) in Down's syndrome is highly responsive to chemotherapy: experience on Pediatric Oncology Group AML Study 8498. Blood 1992;80:2210-4.

50. Zeller B, Gustafsson G, Forestier E, et al. Acute leukaemia in children with Down syndrome: a population-based Nordic study. British journal of haematology 2005;128:797804.

51. Creutzig U, Reinhardt D, Diekamp S, Dworzak M, Stary J, Zimmermann M. AML patients with Down syndrome have a high cure rate with AML-BFM therapy with reduced dose intensity. Leukemia 2005;19:1355-60.

52. Lange BJ, Kobrinsky N, Barnard DR, et al. Distinctive demography, biology, and outcome of acute myeloid leukemia and myelodysplastic syndrome in children with Down syndrome: Children's Cancer Group Studies 2861 and 2891. Blood 1998;91:608-15.

53. Ravindranath Y, Yeager AM, Chang MN, et al. Autologous bone marrow transplantation versus intensive consolidation chemotherapy for acute myeloid leukemia in childhood. Pediatric Oncology Group. N Engl J Med 1996;334:1428-34. 
54. Rao A, Hills RK, Stiller C, et al. Treatment for myeloid leukaemia of Down syndrome: population-based experience in the UK and results from the Medical Research Council AML 10 and AML 12 trials. British journal of haematology 2006;132:576-83.

55. Ravindranath Y, Taub JW. Down syndrome and acute myeloid leukemia. Lessons learned from experience with high-dose Ara-C containing regimens. Advances in experimental medicine and biology 1999;457:409-14.

56. O'Brien MM, Taub JW, Chang MN, et al. Cardiomyopathy in children with Down syndrome treated for acute myeloid leukemia: a report from the Children's Oncology Group Study POG 9421. Journal of clinical oncology : official journal of the American Society of Clinical Oncology 2008;26:414-20.

57. Gamis AS, Woods WG, Alonzo TA, et al. Increased age at diagnosis has a significantly negative effect on outcome in children with Down syndrome and acute myeloid leukemia: a report from the Children's Cancer Group Study 2891. Journal of clinical oncology : official journal of the American Society of Clinical Oncology 2003;21:3415-22.

58. Kudo K, Kojima S, Tabuchi K, et al. Prospective study of a pirarubicin, intermediatedose cytarabine, and etoposide regimen in children with Down syndrome and acute myeloid leukemia: the Japanese Childhood AML Cooperative Study Group. Journal of clinical oncology : official journal of the American Society of Clinical Oncology 2007;25:5442-7.

59. Al-Ahmari A, Shah N, Sung L, Zipursky A, Hitzler J. Long-term results of an ultra lowdose cytarabine-based regimen for the treatment of acute megakaryoblastic leukaemia in children with Down syndrome. British journal of haematology 2006;133:646-8.

60. Tandonnet J, Clavel J, Baruchel A, Nacka F, Perel Y. Myeloid leukaemia in children with Down syndrome: report of the registry-based French experience between 1990 and 2003. Pediatric blood \& cancer 2010;54:927-33.

61. Hasle H, Abrahamsson J, Arola M, et al. Myeloid leukemia in children 4 years or older with Down syndrome often lacks GATA1 mutation and cytogenetics and risk of relapse are more akin to sporadic AML. Leukemia 2008;22:1428-30.

62. Blink M, Zimmermann M, von Neuhoff C, et al. Normal karyotype is a poor prognostic factor in myeloid leukemia of Down syndrome: a retrospective, international study. Haematologica 2014;99:299-307.

63. Loew TM, Gamis A, Smith FO, et al. Down syndrome patients with relapsed acute myelogenous leukemia. Blood 2004;104:Abstract 4526.

64. Taga T, Saito AM, Kudo K, et al. Clinical characteristics and outcome of refractory/relapsed myeloid leukemia in children with Down syndrome. Blood 2012;120:1810-5.

65. Muramatsu H, Sakaguchi H, Taga T, et al. Reduced intensity conditioning in allogeneic stem cell transplantation for AML with Down syndrome. Pediatric blood \& cancer 2014;61:925-7.

66. Caldwell JT, Edwards H, Buck SA, Ge Y, Taub JW. Targeting the wee1 kinase for treatment of pediatric Down syndrome acute myeloid leukemia. Pediatric blood \& cancer 2014.

67. Wen Q, Goldenson B, Silver SJ, et al. Identification of regulators of polyploidization presents therapeutic targets for treatment of AMKL. Cell 2012;150:575-89.

68. Stankov MV, El Khatib M, Kumar Thakur B, et al. Histone deacetylase inhibitors induce apoptosis in myeloid leukemia by suppressing autophagy. Leukemia 2014;28:577-88.

69. Lange B. The management of neoplastic disorders of haematopoiesis in children with Down's syndrome. British journal of haematology 2000;110:512-24.

70. $\quad$ Robison LL. Down syndrome and leukemia. Leukemia 1992;6 Suppl 1:5-7.

71. Hasle H, Clemmensen IH, Mikkelsen M. Risks of leukaemia and solid tumours in individuals with Down's syndrome. Lancet 2000;355:165-9. 
72. Chessells JM, Harrison G, Richards SM, et al. Down's syndrome and acute lymphoblastic leukaemia: clinical features and response to treatment. Archives of disease in childhood 2001;85:321-5.

73. Dordelmann M, Schrappe M, Reiter A, et al. Down's syndrome in childhood acute lymphoblastic leukemia: clinical characteristics and treatment outcome in four consecutive BFM trials. Berlin-Frankfurt-Munster Group. Leukemia 1998;12:645-51.

74. Kalwinsky DK, Raimondi SC, Bunin NJ, et al. Clinical and biological characteristics of acute lymphocytic leukemia in children with Down syndrome. American journal of medical genetics Supplement 1990;7:267-71.

75. Maloney KW. Acute lymphoblastic leukaemia in children with Down syndrome: an updated review. British journal of haematology 2011;155:420-5.

76. Whitlock JA, Sather HN, Gaynon P, et al. Clinical characteristics and outcome of children with Down syndrome and acute lymphoblastic leukemia: a Children's Cancer Group study. Blood 2005;106:4043-9.

77. Bassal M, La MK, Whitlock JA, et al. Lymphoblast biology and outcome among children with Down syndrome and ALL treated on CCG-1952. Pediatric blood \& cancer 2005;44:21-8.

78. Pui CH, Raimondi SC, Borowitz MJ, et al. Immunophenotypes and karyotypes of leukemic cells in children with Down syndrome and acute lymphoblastic leukemia. Journal of clinical oncology : official journal of the American Society of Clinical Oncology 1993;11:13617.

79. Ragab AH, Abdel-Mageed A, Shuster JJ, et al. Clinical characteristics and treatment outcome of children with acute lymphocytic leukemia and Down's syndrome. A Pediatric Oncology Group study. Cancer 1991;67:1057-63.

80. Buitenkamp TD, Izraeli S, Zimmermann M, et al. Acute lymphoblastic leukemia in children with Down syndrome: a retrospective analysis from the Ponte di Legno study group. Blood 2014;123:70-7.

81. Arico M, Ziino 0, Valsecchi MG, et al. Acute lymphoblastic leukemia and Down syndrome: presenting features and treatment outcome in the experience of the Italian Association of Pediatric Hematology and Oncology (AIEOP). Cancer 2008;113:515-21. 82. O'Connor D, Bate J, Wade R, et al. Infection-related mortality in children with acute lymphoblastic leukemia: an analysis of infectious deaths on UKALL2003. Blood 2014;124:1056-61.

83. Maloney KW, Larsen E, Mattano LA, Jr., et al. Improvement in the Infection-related Mortality for Children with Down Syndrome (DS) in Contemporary Children's Oncology Group (COG) Acute Lymphoblastic Leukemia (ALL) Clinical Trials. Pediatric blood \& cancer 2010;54:787-864.

84. Maloney KW, Carroll WL, Carroll AJ, et al. Down syndrome childhood acute lymphoblastic leukemia has a unique spectrum of sentinel cytogenetic lesions that influences treatment outcome: a report from the Children's Oncology Group. Blood 2010;116:1045-50. 85. Hargrave DR, Hann, II, Richards SM, et al. Progressive reduction in treatment-related deaths in Medical Research Council childhood lymphoblastic leukaemia trials from 1980 to 1997 (UKALL VIII, X and XI). British journal of haematology 2001;112:293-9.

86. Izraeli S, Vora A, Zwaan CM, Whitlock J. How I treat ALL in Down's syndrome: pathobiology and management. Blood 2014;123:35-40.

87. Garre ML, Relling MV, Kalwinsky D, et al. Pharmacokinetics and toxicity of methotrexate in children with Down syndrome and acute lymphocytic leukemia. The Journal of pediatrics 1987;111:606-12.

88. Peeters MA, Rethore MO, Lejeune J. In vivo folic acid supplementation partially corrects in vitro methotrexate toxicity in patients with Down syndrome. British journal of haematology 1995;89:678-80. 
89. Taub JW, Ge Y. Down syndrome, drug metabolism and chromosome 21. Pediatric blood \& cancer 2005;44:33-9.

90. Matloub Y, Bostrom BC, Hunger SP, et al. Escalating intravenous methotrexate improves event-free survival in children with standard-risk acute lymphoblastic leukemia: a report from the Children's Oncology Group. Blood 2011;118:243-51.

91. Maloney KW, Larsen E, Devidas M, et al. Event Free (EFS) and Overrall Survival (OS) for Children with Down Syndrome (DS) and B-Lymphoblastic Leukemia (B-ALL) in Children's Oncology Group (COG) Clinical Trails AALL0232 and AALL0331. Pediatric blood \& cancer 2014;61:S1-S104.

92. Meyr F, Escherich G, Mann G, et al. Outcomes of treatment for relapsed acute lymphoblastic leukaemia in children with Down syndrome. British journal of haematology 2013;162:98-106.

93. Hertzberg L, Vendramini E, Ganmore I, et al. Down syndrome acute lymphoblastic leukemia, a highly heterogeneous disease in which aberrant expression of CRLF2 is associated with mutated JAK2: a report from the International BFM Study Group. Blood 2010;115:100617.

94. Mullighan CG, Collins-Underwood JR, Phillips LA, et al. Rearrangement of CRLF2 in Bprogenitor- and Down syndrome-associated acute lymphoblastic leukemia. Nature genetics 2009;41:1243-6.

95. Cario G, Zimmermann M, Romey R, et al. Presence of the P2RY8-CRLF2 rearrangement is associated with a poor prognosis in non-high-risk precursor B-cell acute lymphoblastic leukemia in children treated according to the ALL-BFM 2000 protocol. Blood 2010;115:53937.

96. Bercovich D, Ganmore I, Scott LM, et al. Mutations of JAK2 in acute lymphoblastic leukaemias associated with Down's syndrome. Lancet 2008;372:1484-92.

97. Mullighan CG, Zhang J, Harvey RC, et al. JAK mutations in high-risk childhood acute lymphoblastic leukemia. Proceedings of the National Academy of Sciences of the United States of America 2009;106:9414-8.

98. Harvey RC, Mullighan CG, Chen IM, et al. Rearrangement of CRLF2 is associated with mutation of JAK kinases, alteration of IKZF1, Hispanic/Latino ethnicity, and a poor outcome in pediatric B-progenitor acute lymphoblastic leukemia. Blood 2010;115:5312-21.

99. Buitenkamp TD, Pieters R, Gallimore NE, et al. Outcome in children with Down's syndrome and acute lymphoblastic leukemia: role of IKZF1 deletions and CRLF2 aberrations. Leukemia 2012;26:2204-11. 